\title{
Flavonoid intake in relation to cognitive function in later life in the Lothian Birth Cohort 1936
}

\author{
Catherine Butchart ${ }^{1 *}$, Janet Kyle ${ }^{2}$, Geraldine McNeill ${ }^{2}$, Janie Corley ${ }^{3}$, Alan J. Gow ${ }^{3,4}$, John M. Starr ${ }^{3,4,5}$ \\ and Ian J. Deary ${ }^{3,4}$ \\ ${ }^{1}$ Department of Medicine for the Elderly, Woodend Hospital, Eday Road, Aberdeen AB15 6XS, UK \\ ${ }^{2}$ Department of Applied Health Sciences, University of Aberdeen, Aberdeen, UK \\ ${ }^{3}$ Department of Psychology, University of Edinburgh, Edinburgh, UK \\ ${ }^{4}$ Centre for Cognitive Ageing and Cognitive Epidemiology, University of Edinburgh, Edinburgh, UK \\ ${ }^{5}$ Geriatric Medicine Unit, University of Edinburgh, Edinburgh, UK
}

(Received 7 June 2010 - Revised 12 November 2010 - Accepted 10 December 2010 - First published online 8 February 2011)

\section{Abstract}

Previous studies have suggested a link between flavonoid intake and better cognitive function in later life but have not been able to control for possible confounding by prior intelligence quotient (IQ). The aim of the present study was to address this issue in a cross-sectional survey of 1091 men and women born in 1936, in whom IQ was measured at age 11 years. At the age of 70 years, participants carried out various neuropsychological tests and completed a FFQ. Associations between test scores and nutrient intake were assessed by linear regression with adjustment for potentially confounding variables. Total fruit, citrus fruits, apple and tea intakes were initially found to be associated with better scores in a variety of cognitive tests, but the associations were no longer statistically significant after adjusting for confounding factors, including childhood IQ. Flavanone intake was initially found to be associated with better scores in verbal fluency $(P=0.003$, with standardised regression coefficient $0 \cdot 10)$, but, again, the association was no longer statistically significant after adjusting for confounding factors. These findings do not support a role for flavonoids in the prevention of cognitive decline in later life. Studies of diet and cognitive function should include measurement of potential confounding variables, including prior IQ wherever possible.

\section{Key words: Cognition: Elderly: Flavonoids}

The proportion of the population aged over 65 years is steadily increasing, and the demographics of the world's population are predicted to change fairly rapidly over the next 20 years. The United Nations Human Development Report predicts that the proportion of the population aged 65 years and older in the UK will rise from 13.8 to $18.1 \%$ between 2003 and 2015. In the USA, the predicted rise is from $10 \cdot 7$ to $14.1 \%$, and one of the largest rises predicted is in Singapore, where it is expected to increase from 6.5 to $13.3 \%{ }^{(1)}$. These changes are likely to have major social and economic impacts, together with a shift in the cognitive profile of populations worldwide. Cognitive ageing is one example of why these population changes are relevant. Age-related cognitive changes are very variable between individuals, but in a significant proportion of the population, they lead to impairments of function in activities of daily living. At the most severe end of these changes, the UK Alzheimer's Society predicts that the number of people in the UK with dementia will rise from the current estimate of 700000 to 940000 in $2021^{(2)}$. There are potentially high social and economic costs resulting from the provision of care for these individuals. Hence, any means by which cognitive decline may be prevented or reduced at a population level are becoming increasingly important.

Oxidative stress, neuroinflammation and impaired neurogenesis are mechanisms that are thought to contribute to neuronal loss and cognitive decline ${ }^{(3)}$. As a result, there has been a great deal of interest in dietary and therapeutic interventions that might prevent damage to the central nervous system from these processes. Dietary flavonoids are an example of naturally occurring antioxidant compounds and are claimed to confer a protective effect against cognitive

Abbreviations: IQ, intelligence quotient; NART, National Adult Reading Test; WAIS, Wechsler Adult Intelligence Scale-III UK; WMS, Wechsler Memory ScaleIII UK. 
decline $^{(3)}$. Although rapidly metabolised in the body, flavonoid metabolites have been found in the brain at concentrations capable of modulating intracellular signalling cascades, leading to protection against neuronal loss ${ }^{(4)}$. Flavonoids are found in a wide variety of plant-based foods and drinks, particularly fruit, vegetables, tea, red wine and cocoa. They can be divided into six different families: flavonols, flavanols, flavones, flavanones, anthocyanidins and isoflavones, with several additional minor subclasses ${ }^{(5)}$. Each group is found in different types of food and drink, and in varying quantities $^{(6)}$. The primary dietary sources of flavonols and flavanols are tea, apples, red wine and chocolate, as well as a range of other fruit and vegetables. Citrus fruits and wine are the main sources of flavanones, while sweet pepper, lettuce and herbs found in processed foods are the main dietary contributors of flavones ${ }^{(6)}$.

In recent years, there has been a great deal of interest in the potential effects of dietary flavonoids on health. In addition to their antioxidant properties, flavonoids have also been reported to have anti-inflammatory properties, beneficial effects on cardiovascular health and also perhaps help to

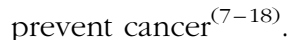

A number of studies have suggested a possible association between higher flavonoid intake and a lower risk of dementia $^{(19-22)}$. However, cross-sectional studies may not be able to take into account the possibility that prior education, intelligence quotient (IQ) or socio-economic status (all of which are strongly associated) may influence both later diet and cognitive performance, leading to a potentially spurious association between diet and cognitive performance in later life. Batty et $a l .{ }^{(23)}$ reported that children with higher mental ability scores reported significantly higher consumption of fruit and vegetables than those with lower cognitive ability scores. Therefore, an optimal design would include these variables in any analysis attempting to correlate flavonoid intake and cognition in old age. This possibility has also been highlighted by a recent study of UK civil servants, which has found that associations between dietary patterns and cognitive ability were substantially attenuated by adjustment for educational attainment, suggesting that education (or perhaps the cognitive ability level that preceded it) influences not only cognition in later life but dietary patterns as well ${ }^{(24)}$

The Lothian Birth Cohort 1936 is a longitudinal study of cognitive ageing in which information on cognitive ability before cognitive ageing is available from IQ tests carried out at age 11 years. We used this population to investigate the potential role of different subclasses of flavonoids in reducing cognitive decline, after adjusting for prior cognitive ability and other potential confounding variables. The other variables included were age, sex, smoking status, socio-economic group, years of full-time education and presence/absence of the apoE $\varepsilon 4$ allele. Smoking was included as a confounding factor, given its association with vascular disease (such as stroke), which in turn can affect cognitive function. The apoE $\varepsilon 4$ allele status was included in view of the association with normal as well as pathological cognitive ageing ${ }^{(25,26)}$.

\section{Materials and methods}

\section{Participants}

The Lothian Birth Cohort 1936 consists of 1091 relatively healthy men and women born in 1936 living independently in and around Edinburgh. They had cognitive function measured at mean ages of 11 and 70 years. Further details of the profile of the cohort, including the methods used for tracing the participants, have been reported fully elsewhere ${ }^{(27)}$. The participants were invited to attend for detailed cognitive, biomedical and psychosocial testing between 2004 and 2007 , at a mean age of 70 years. Socio-economic class was determined using the main lifetime occupation of the participant (or, if required, the spouse) using the UK Registrar General's classification of occupation ${ }^{(28)}$. Earlier studies involving the Lothian Birth Cohort 1936 have extensively examined childhood socio-economic status and social mobility within the cohort ${ }^{(29,30)}$. Childhood social circumstances did not make any contribution to cognitive ability in old age, whereas adult occupational social class did; therefore, only the latter was included in the present study. The present study was conducted according to the guidelines laid down in the Declaration of Helsinki, and all procedures involving human subjects were approved by the Multi-Centre Research Ethics Committee for Scotland (MREC/01/0/56) and the Lothian Research Ethics Committee (LREC/2003/2/29). All participants gave their written informed consent.

\section{FFQ}

At the clinic visit, the participants were given version 7.0 of the Scottish Collaborative Group FFQ, with verbal and written instructions for completion at home. This semi-quantitative FFQ was previously developed for use in older adults as a modified version of the Scottish Collaborative Group FFQ version 6.4, used in younger adults (see www.foodfrequency.org.uk). The FFQ version 7.0 lists 168 foods or drinks, each with an appropriate household measure, such as 'one slice', for the estimation of portion size. Participants were asked to select one of nine possible responses for the typical amount and frequency of each food consumed in the previous 2-3 months. These FFQ were administered between 2004 and 2007. They were completed by the participants at home and returned by mail, and were checked on return for completeness. Participants with missing or ambiguous responses were contacted by letter to supply the required information where possible. Of these questionnaires, ninety-eight were not returned, twenty-six were returned blank and thirty-nine had more than ten missing values (after checking and re-contact) and were therefore excluded from analysis. A further fortysix questionnaires were excluded as having extreme energy intakes (defined as $<2.5$ th or $>97.5$ th centile for energy intake), leaving 882 questionnaires for analysis. Intake of the flavonoid subclasses was estimated using a UK flavonoids database, which includes 396 items $^{(6)}$. Intake of flavonoids was adjusted for energy using the residual method ${ }^{(31)}$. Intake of flavonoid-rich foods and drinks was also analysed. 
Table 1. Participant characteristics in the Lothian Birth Cohort 1936 study (Numbers and percentages (for categorical variables) and mean values and standard deviations (for continuous variables))

\begin{tabular}{|c|c|c|c|c|}
\hline & \multicolumn{2}{|c|}{$\begin{array}{l}\text { Included } \\
\text { (n 882) }\end{array}$} & \multicolumn{2}{|c|}{$\begin{array}{l}\text { Not included } \\
\quad(n \text { 209) }\end{array}$} \\
\hline & $n$ & $\%$ & $n$ & $\%$ \\
\hline \multicolumn{5}{|l|}{ Sex } \\
\hline Male & 425 & 48.2 & 123 & $58 \cdot 9$ \\
\hline Female & 457 & $51 \cdot 8$ & 86 & $41 \cdot 1$ \\
\hline \multicolumn{5}{|l|}{ Years of full-time education } \\
\hline Mean & \multicolumn{2}{|c|}{$10 \cdot 8$} & \multicolumn{2}{|c|}{$10 \cdot 5^{\star \star}$} \\
\hline SD & \multirow{2}{*}{\multicolumn{2}{|c|}{$1 \cdot 2$}} & \multirow{2}{*}{\multicolumn{2}{|c|}{$1 \cdot 1$}} \\
\hline Social class (based on occupation) & & & & \\
\hline 1 (Professional) & 160 & 18.5 & $30^{\star \star}$ & $14 \cdot 6$ \\
\hline 2 (Managerial/intermediate) & 335 & 38.8 & $67^{\star *}$ & $32 \cdot 7$ \\
\hline 3 (Skilled non-manual) & 205 & 23.7 & $41^{\star \star}$ & $20 \cdot 0$ \\
\hline 3 (Skilled manual) & 135 & $15 \cdot 6$ & $52^{\star *}$ & $25 \cdot 4$ \\
\hline 4 or 5 (Partly or unskilled manual) & 29 & 3.4 & $15^{\star *}$ & $7 \cdot 3$ \\
\hline \multicolumn{5}{|l|}{ Current smoking status } \\
\hline Smoker & 107 & $12 \cdot 1$ & 37 & $17 \cdot 7$ \\
\hline Ex-smoker & 383 & 43.4 & 90 & $43 \cdot 1$ \\
\hline Never smoker & 392 & 44.4 & 82 & $39 \cdot 2$ \\
\hline \multicolumn{5}{|l|}{ IQ at age 11 years* } \\
\hline Mean & \multicolumn{2}{|c|}{101.4} & \multicolumn{2}{|c|}{$93 \cdot 8^{\star *}$} \\
\hline SD & \multicolumn{2}{|c|}{$14 \cdot 0$} & \multicolumn{2}{|c|}{$7 \cdot 3$} \\
\hline \multirow{2}{*}{\multicolumn{5}{|c|}{$\begin{array}{l}\text { Later adulthood cognitive test scores } \\
\qquad \mathrm{IQ}^{\star}\end{array}$}} \\
\hline & & & & \\
\hline Mean & \multicolumn{2}{|c|}{$101 \cdot 6$} & \multicolumn{2}{|c|}{$92 \cdot 9^{* *}$} \\
\hline SD & \multirow{2}{*}{\multicolumn{2}{|c|}{$13 \cdot 4$}} & \multicolumn{2}{|c|}{$18 \cdot 9$} \\
\hline \multicolumn{3}{|l|}{ MMSE† } & & \\
\hline Mean & \multicolumn{2}{|c|}{$\begin{array}{c}28.9 \\
1.4\end{array}$} & \multicolumn{2}{|c|}{$28 \cdot 2^{* \star}$} \\
\hline SD & \multicolumn{2}{|c|}{$1 \cdot 4$} & \multicolumn{2}{|c|}{$1 \cdot 8$} \\
\hline \multicolumn{5}{|l|}{ NART $\ddagger$} \\
\hline Mean & \multicolumn{2}{|c|}{$35 \cdot 2$} & \multicolumn{2}{|c|}{$31.5^{\star \star}$} \\
\hline SD & \multicolumn{2}{|c|}{$7 \cdot 7$} & 9 . & \\
\hline Verbal fluency§ & & & & \\
\hline Mean & & & 39. & \\
\hline SD & & & 12 & \\
\hline ApoE $\varepsilon 4$ allele present & 243 & $29 \cdot 0$ & 63 & 33.0 \\
\hline
\end{tabular}

$I Q$, intelligence quotient; MMSE, mini-mental state examination; NART, National Adult Reading Test.

Values were significantly different $\left({ }^{\star *} P<0.01\right)$.

* Test of general intelligence. Both IQ at age 11 years and IQ at age 70 years were standardised to a mean value of 100 (SD 15).

† Screening test for possible dementia.

$\ddagger$ Marker of peak prior intelligence.

$\S$ Test of executive function.

The repeatability and validity of the FFQ for other nutrients was assessed in a separate group of eighty-three men and women aged 68 years or over, who completed the FFQ version 7.0 and a $4 \mathrm{~d}$ weighed diet diary ${ }^{(32)}$. In these participants, the Spearman rank correlation values for energy-adjusted flavonoid intake from food by the FFQ and diet diary were $0.52,0.61$ and 0.61 for flavonols, procyanidins and catechins, respectively, at a significance level of $P<0 \cdot 01$. Flavone and flavanone correlation coefficient values were weaker, at 0.26 $(P<0.05)$ and $0.47(P<0.01)$, respectively ( $\mathrm{J}$. Kyle, unpublished results).

\section{Cognitive function}

Full details of and individual references for the cognitive tests carried out at age 70 years have been provided in an earlier open access publication ${ }^{(27)}$. These included the Moray
House Test, which is a test of general intelligence based mainly on verbal reasoning. This was also administered at age 11 years. The Moray House Test is used as a test for IQ. Other tests included the mini-mental state examination, which is a brief screening test for possible dementia; the National Adult Reading Test (NART), which estimates peak prior intelligence and is based on the pronunciation of fifty irregular words; and verbal fluency, which is a test of executive function and involves saying as many words as possible beginning with the letters $\mathrm{C}, \mathrm{F}$ and $\mathrm{L}$. Participants also took non-verbal subtests from the Wechsler Adult Intelligence Scale-III UK (WAIS) and a number of memory tests from the Wechsler Memory Scale-III UK (WMS). For the purpose of data reduction, the WMS and WAIS subtest scores were grouped into those which related to memory (logical memory I and II, spatial span forward/backward, verbal paired associates I and II, letter-number sequencing and backwards digit span) and general fluid intelligence (matrix reasoning, block design, letter-number sequencing, backward digit span, symbol search and digit symbol coding). Principal component analysis of the WMS and WAIS subtests' scores was carried out. In each case, there was a first unrotated principal component, which accounted for a large proportion of the variance and on which all subtests had high loadings. Therefore, subjects were given scores on these to give a 'memory factor' and 'general intelligence factor'. In a similar fashion, a 'processing speed factor' score was generated. The principal component analysis for this included symbol search and digit symbol coding subtests from the WAIS, and also mean scores on simple reaction time and four-choice reaction time, and inspection time (a psychophysical task assessing speed of the early stages of visual processing) $)^{(33,34)}$.

\section{Statistical analysis}

The associations between flavonoid intake and cognitive test scores were assessed using multiple linear regression, with the cognitive test scores as the dependent variable and the energy-adjusted flavonoid intake as the independent variable. Flavonoid intake in $\mathrm{mg} / \mathrm{d}$ was used rather than categories of intake, in order to retain more information regarding the vari-

Table 2. Reported frequency of consumption of flavonoid-rich foods in study participants who consume the foodstuff listed in the questionnaire

\begin{tabular}{lccl}
\hline & $\begin{array}{c}\text { Participants } \\
(n)\end{array}$ & $\begin{array}{c}\text { Consumers } \\
(\%)^{*}\end{array}$ & $\begin{array}{c}\text { Frequency } \\
(\text { mode })\end{array}$ \\
\hline Total fruit† & 882 & 99 & $2-3 / \mathrm{d}$ \\
Apples & 882 & 81 & $2-3 /$ week \\
Citrus fruits & 882 & 81 & $2-3 /$ week \\
Total vegetables $\ddagger$ & 882 & 100 & $2-3 / \mathrm{d}$ \\
Tea & 882 & 85 & $2-3 / \mathrm{d}$ \\
Red wine & 882 & 55 & $1-3 /$ week \\
Red wine drinkers only & 488 & 100 & $2-3 /$ week \\
Chocolate§ & 882 & 84 & $2-3 /$ week \\
\hline
\end{tabular}

* Percentage of cohort who consume.

†Total number of measures of fruit as defined on the FFQ.

$¥$ Total number of measures of vegetables as defined on the FFQ.

$\S$ Total number of measures of chocolate bars, sweets and biscuits reported. 
Table 3. Dietary flavonoid intake (mg/d) in the Lothian Birth Cohort 1936 study (Mean values with their standard errors, medians and interquartile ranges (IQR))

\begin{tabular}{lccrrrc}
\hline & Participants & Consumers* $(\%)$ & Mean & SEM & Median & IQR \\
\hline Flavonols & 882 & 100 & 37.7 & 0.9 & 35.8 & $16.0-63.5$ \\
Flavones & 882 & 100 & 0.3 & 0.0 & 0.2 & $0.2-0.4$ \\
Catechins & 882 & 100 & 186.3 & 4.8 & 181.6 & $69.6-349.9$ \\
Procyanidins & 882 & 100 & 52.7 & 1.2 & 48.5 & $22.3-85.7$ \\
Flavanones & 882 & 98 & 25.2 & 0.9 & 17.5 & $10 \cdot 0-38 \cdot 1$ \\
\hline
\end{tabular}

*Percentage of cohort who consume.

ation in flavonoid intake from the FFQ. The data are therefore reported as standardised regression coefficients. This represents the change in the standard deviation of a cognitive test score per $\mathrm{mg}$ increase in flavonoid intake/d. This method was used in order to standardise across all cognitive tests. We used three regression models. Model 1 was a basic model, which included the energy-adjusted flavonoid intake plus sex and exact age (in days) at testing in later life. This model was designed to demonstrate crude associations between flavonoid intake and cognitive performance in later life. Model 2 also included IQ at age 11 years as an additional covariate, to adjust for any influence of early-life cognitive ability on the association between flavonoid intake and cognition in later life. This approach was used in preference to using change in IQ between the two time points, as it retains the information on both initial and final absolute values. Model 3 additionally included other potential confounding variables: smoking status (current, ex- or never-smoker); social class; years of full-time education; presence/absence of the apoE $\varepsilon 4$ allele.

Associations between intake of flavonoid-rich foods/drinks (total fruit intake, total vegetable intake, apples, citrus fruits, tea, chocolate and red wine) and cognitive test scores were analysed in the same manner. All analyses were carried out using SPSS version 16 (SPSS, Inc., Chicago, IL, USA).

\section{Results}

The characteristics of the study participants included in the analysis are shown in Table 1, along with those not included in the analyses, for comparison. In both groups, participants of lower socio-economic status were under-represented. Those

Table 4. Association between intake of flavonoid-rich foods and intelligence quotient (IQ) at age 11 years in the Lothian Birth Cohort 1936 study

(Correlation coefficients and $P$ values)

\begin{tabular}{lcc}
\hline & \multicolumn{2}{c}{ IQ at age 11 years } \\
\cline { 2 - 3 } Food/drink & Correlation coefficient & $P$ \\
\hline Chocolate & 0.10 & 0.003 \\
Total fruit & 0.05 & 0.164 \\
Total vegetables & 0.07 & 0.051 \\
Tea & 0.12 & 0.001 \\
Apples & 0.02 & 0.474 \\
Citrus fruits & 0.02 & 0.512 \\
Red wine & 0.26 & 0.0001 \\
\hline
\end{tabular}

*Spearman's rank correlation coefficient. included in the analysis were more likely to be from higher socio-economic groups and to have higher childhood IQ and higher cognitive test scores in later life than those who were not included.

Tables 2 and 3 describe the intake of flavonoid-rich foods and of the different flavonoid subgroups in the participants. Tables 4 and 5 show the associations between intake of flavonoid-rich foods/flavonoids and IQ at age 11 years. There was a significant positive association between IQ at age 11 years and intake of chocolate and red wine. Conversely, there was a significant negative association between IQ at age 11 years and intake of tea, flavonols, catechins and procyanidins.

Table 6 shows the results of the regression analyses for flavonoid-rich foods. Total fruit intake was significantly associated with higher scores in the NART after adjusting for age, sex and childhood IQ. However, after adjusting for smoking, socio-economic status, education and the apoE $\varepsilon 4$ allele in model 3, the association was no longer statistically significant at the $5 \%$ level. A similar pattern was observed for citrus fruits and red wine with NART scores. Citrus fruit intake was also associated with significantly higher scores in the mini-mental state examination, verbal fluency and speed tests, but this association was no longer statistically significant after adjusting for childhood IQ in model 2.

Tea intake was associated with lower scores in the later-life IQ, NART, verbal fluency, speed and general intelligence tests in model 1, but, again, the association was no longer statistically significant after adjusting for childhood IQ in model 2. These findings are similar to those reported by Corley et $a{ }^{(34)}$. In that study, IQ at age 11 years was found to account for most of the observed positive associations between caffeine consumption and cognitive ability at age 70 years. A similar pattern was observed in the present

Table 5. Association between flavonoid intake and intelligence quotient (IQ) at age 11 years in the Lothian Birth Cohort 1936 study (Correlation coefficient values and $P$ values)

\begin{tabular}{lcc}
\hline & \multicolumn{2}{c}{ IQ at age 11 years } \\
\cline { 2 - 3 } Nutrient & Correlation coefficient* & $P$ \\
\hline Flavonols & -0.11 & 0.001 \\
Flavones & 0.001 & 0.973 \\
Catechins & -0.12 & 0.001 \\
Procyanidins & -0.08 & 0.014 \\
Flavanones & 0.06 & 0.081 \\
\hline
\end{tabular}

*Spearman's rank correlation coefficient. 
Table 6. Association between intake of flavonoid-rich foods and cognitive test scores at age 70 years in the Lothian Birth Cohort 1936 study

(Standardised regression coefficients)

\begin{tabular}{|c|c|c|c|c|c|c|c|}
\hline Model† & 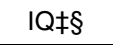 & MMSE§ & NART§ & VF§ & Memory§ & Speed§ & GI§ \\
\hline \multicolumn{8}{|c|}{ Total fruit } \\
\hline 1 & 0.01 & 0.06 & $0.07^{\star}$ & 0.05 & 0.01 & 0.04 & 0.04 \\
\hline 2 & -0.01 & 0.05 & $0.05^{\star}$ & 0.05 & -0.01 & 0.03 & 0.02 \\
\hline 3 & -0.03 & 0.03 & 0.02 & 0.05 & -0.02 & 0.01 & -0.01 \\
\hline \multicolumn{8}{|c|}{ Total vegetables } \\
\hline 1 & $0.08^{\star}$ & 0.05 & $0.09^{*}$ & $0.08^{*}$ & 0.01 & 0.03 & 0.05 \\
\hline 2 & 0.04 & 0.03 & 0.05 & 0.06 & -0.03 & 0.01 & 0.02 \\
\hline 3 & 0.01 & 0.02 & 0.01 & 0.05 & -0.04 & -0.01 & 0.01 \\
\hline \multicolumn{8}{|l|}{ Apples } \\
\hline 1 & -0.01 & 0.01 & $0.07^{*}$ & 0.02 & -0.01 & 0.04 & 0.01 \\
\hline 2 & -0.01 & 0.02 & $0.07^{*}$ & 0.03 & -0.01 & 0.05 & 0.01 \\
\hline 3 & -0.03 & 0.01 & $0.05^{*}$ & 0.04 & -0.02 & 0.02 & -0.01 \\
\hline \multicolumn{8}{|c|}{ Citrus fruits } \\
\hline 1 & 0.05 & $0.07^{\star}$ & $0 \cdot 12^{\star \star}$ & $0.08^{*}$ & 0.03 & $0.07^{\star}$ & 0.04 \\
\hline 2 & 0.01 & 0.05 & $0.07^{\star \star}$ & 0.06 & -0.01 & 0.05 & -0.01 \\
\hline 3 & -0.01 & 0.03 & 0.05 & 0.05 & -0.01 & 0.04 & -0.01 \\
\hline \multicolumn{8}{|l|}{ Tea } \\
\hline 1 & $-0.13^{\star \star}$ & -0.02 & $-0 \cdot 12^{\star \star}$ & $-0 \cdot 10^{\star \star}$ & -0.04 & $-0.11^{*}$ & $-0.12^{\star \star}$ \\
\hline 2 & -0.01 & 0.05 & -0.02 & -0.04 & 0.04 & -0.04 & -0.02 \\
\hline 3 & -0.01 & 0.05 & 0.01 & -0.03 & 0.04 & -0.06 & -0.03 \\
\hline \multicolumn{8}{|c|}{ Red wine } \\
\hline 1 & $0.18^{\star *}$ & 0.04 & $0.23^{\star \star}$ & $0 \cdot 10^{\star *}$ & $0 \cdot 14^{\star *}$ & $0.11^{\star \star}$ & $0 \cdot 18^{\star *}$ \\
\hline 2 & 0.03 & -0.05 & $0.09^{\star \star}$ & 0.03 & 0.03 & 0.02 & $0.06^{\star}$ \\
\hline 3 & -0.01 & $-0.08^{\star}$ & 0.04 & 0.03 & -0.01 & -0.03 & 0.01 \\
\hline \multicolumn{8}{|c|}{ Chocolate } \\
\hline 1 & $0.08^{\star}$ & 0.06 & -0.01 & 0.02 & 0.04 & $0 \cdot 12^{\star \star}$ & $0.08^{*}$ \\
\hline 2 & 0.01 & 0.03 & $-0.07^{\star}$ & -0.01 & -0.02 & $0.09^{* *}$ & 0.03 \\
\hline 3 & -0.01 & 0.03 & $-0.09^{\star *}$ & 0.01 & -0.03 & $0.08^{\star \star}$ & 0.03 \\
\hline
\end{tabular}

IQ, intelligence quotient (using the Moray House Test); MMSE, mini-mental state examination; NART, National Adult Reading Test; VF, verbal fluency; GI, general intelligence.

${ }^{*} P<0.05,{ }^{* *} P<0.01$

† Model 1: energy-adjusted nutrient intake, age and sex; model 2: as model 1 plus IQ at age 11 years from the Moray House Test; model 3: as model 2 plus smoking, socio-economic group, years of full-time education and presence/ absence of the apoE $\varepsilon 4$ allele.

$\ddagger$ Adjusted for exact age at testing.

$\S I Q$, marker of GI; MMSE, screening tool for possible dementia; NART, marker of peak prior intelligence; VF, test of executive function; memory, speed and GI, overall markers of memory, processing speed and GI.

study for the positive association between total vegetable intake and higher scores in later-life IQ, NART and verbal fluency tests.

The intake of apples was associated with higher scores in the NART, and this remained statistically significant after adjusting for all three models. Chocolate intake was associated with lower scores in the NART but higher scores in speed after adjusting for all three models.

The associations between intake of individual flavonoid groups and test scores are shown in Table 7. Flavanones were associated with higher scores in the NART, and this remained statistically significant after the adjustments made in all three models. They were also associated with higher scores in verbal fluency, but this was no longer statistically significant after adjusting for other covariates in model 3 . Flavonol intake was associated with lower scores in the laterlife IQ, NART, verbal fluency, speed and general intelligence tests in model 1, but this association was no longer statistically significant after adjusting for confounding factors in models 2 and 3 . The same can be said for catechin intake. Procyanidin intake was associated with lower scores in later-life IQ, speed and general intelligence tests in model 1, but, again, this was no longer significant after adjustment in models 2 and 3 .

\section{Discussion}

The results of the present study confirm earlier reports that intake of flavonoid-rich foods in later-life is associated with better cognitive performance in later-life. In particular, flavanone intake, total fruit, total vegetable, citrus fruits, apple, red wine and chocolate intakes were found to be associated with higher cognitive test scores. However, once the data were adjusted for socio-economic status and prior IQ, among other confounding factors, the results were mainly no longer statistically significant. This suggests that some of the observed association may be explained by an influence of prior IQ on diet in later-life (or, indeed, some unknown factor that influences both early-life IQ and diet in later life) rather than an influence of diet on later-life cognition. It is possible that food choices throughout life are influenced by stable trait intelligence, perhaps via its associations with education and subsequent adult occupational social class ${ }^{(23)}$. For example, more intelligent individuals may be more likely to read information regarding healthy lifestyle choices and therefore follow a healthier diet. Such differences in dietary habits might persist into later life. Further support for this theory is provided by the associations that were found between 
Table 7. Association between flavonoid intake from food and cognitive test scores at age 70 years in the Lothian Birth Cohort 1936 study

(Standardised regression coefficients)

\begin{tabular}{|c|c|c|c|c|c|c|c|}
\hline Model† & $\mathrm{IQ} \ddagger \S$ & MMSE§ & NART§ & VF§ & Memory§ & Speed§ & GI§ \\
\hline \multicolumn{8}{|c|}{ Flavonols } \\
\hline 1 & $-0.12^{\star \star}$ & -0.02 & $-0 \cdot 10^{\star \star}$ & $-0.08^{*}$ & -0.04 & $-0.10^{\star \star}$ & $-0.11^{\star \star}$ \\
\hline 2 & -0.01 & 0.04 & 0.01 & -0.02 & 0.04 & -0.03 & -0.02 \\
\hline 3 & -0.01 & 0.05 & 0.02 & -0.02 & 0.04 & -0.05 & -0.03 \\
\hline \multicolumn{8}{|c|}{ Flavones } \\
\hline 1 & 0.02 & 0.06 & -0.01 & -0.03 & -0.03 & 0.05 & 0.02 \\
\hline 2 & 0.05 & 0.06 & 0.02 & -0.02 & -0.01 & 0.06 & 0.04 \\
\hline 3 & 0.02 & 0.06 & -0.01 & -0.05 & -0.02 & 0.03 & 0.03 \\
\hline \multicolumn{8}{|c|}{ Catechins } \\
\hline 1 & $-0.13^{\star *}$ & -0.03 & $-0.11^{\star *}$ & $-0.09^{\star *}$ & -0.03 & $-0.11^{\star *}$ & $-0.12^{\star \star}$ \\
\hline 2 & -0.01 & 0.04 & -0.01 & -0.03 & 0.04 & -0.05 & -0.03 \\
\hline 3 & -0.01 & 0.04 & 0.02 & -0.02 & 0.05 & -0.06 & -0.03 \\
\hline \multicolumn{8}{|c|}{ Procyanidins } \\
\hline 1 & $-0.10^{\star \star}$ & -0.03 & -0.06 & $-0.07^{\star}$ & -0.02 & $-0.10^{\star \star}$ & $-0 \cdot 10^{\star \star}$ \\
\hline 2 & -0.01 & 0.03 & 0.02 & -0.01 & 0.04 & -0.04 & -0.02 \\
\hline 3 & -0.01 & 0.04 & 0.04 & -0.01 & 0.05 & -0.06 & -0.03 \\
\hline \multicolumn{8}{|c|}{ Flavanones } \\
\hline 1 & $0.07^{*}$ & 0.06 & $0 \cdot 14^{\star *}$ & $0.10^{* *}$ & 0.04 & $0.08^{*}$ & $0.07^{*}$ \\
\hline 2 & 0.01 & 0.03 & $0.08^{\star *}$ & $0.07^{\star}$ & -0.01 & 0.06 & 0.02 \\
\hline 3 & -0.01 & 0.02 & $0.05^{\star}$ & 0.06 & -0.01 & 0.04 & 0.01 \\
\hline
\end{tabular}

$I Q$, intelligence quotient (using the Moray House Test); MMSE, mini-mental state examination; NART, National Adult Reading Test; VF, verbal fluency; GI, general intelligence.

${ }^{\star} P<0.05,{ }^{\star \star} P<0.01$.

† Model 1: energy-adjusted nutrient intake, age and sex; model 2: as model 1 plus IQ at age 11 years from the Moray House Test; model 3: as model 2 plus smoking, socio-economic group, years of full-time education and presence/absence of the apoE $\varepsilon 4$ allele.

$\ddagger$ Adjusted for exact age at testing

$\S \mathrm{IQ}$, marker of Gl; MMSE, screening tool for possible dementia; NART, marker of peak prior intelligence; VF, test of executive function; memory, speed and Gl, overall markers of memory, processing speed and GI.

intake of certain flavonoids/flavonoid-rich foods and IQ at age 11 years. This is particularly interesting as other studies have not had access to these early-life cognitive ability data, and, as such, an important confounding factor may have been missed. It has already been reported that childhood IQ accounts for about $50 \%$ of the variance in cognitive test scores in later-life. Furthermore, early-life IQ is said to provide a more accurate estimate of cognitive ability trait levels throughout adult life than IQ at age 70 years. This is because later-life IQ can be affected by cognitive decline in addition to the trait level ${ }^{(35)}$. If prior IQ does indeed also influence dietary patterns ${ }^{(23)}$, then it should be included in studies assessing associations between diet and cognition wherever possible.

Commenges et al. ${ }^{(19)}$ found that higher flavonoid intake was associated with a lower relative risk of dementia, and the same cohort was later found to have a slower decline in mini-mental state examination scores with higher flavonoid intake ${ }^{(21)}$. Interestingly, those with higher flavonoid intake also had higher mini-mental state examination scores at baseline, but the investigators did not control for energy intake, prior IQ or socio-economic status. Nurk et $a l^{(22)}$ found that tea, chocolate and wine consumers performed better in a variety of cognitive tests compared with non-consumers. Again, the investigators did not adjust for prior IQ or socio-economic status.

In the present study, several associations were found between diet and cognitive function. Apple intake and flavanone intake were associated with significantly higher scores in the NART after adjusting for confounding factors. However, the NART is said to represent crystallised intelligence and to be a marker of pre-morbid or peak prior intelligence. The NART, therefore, is likely to be indicating prior intelligence in adulthood, at an age later than that assessed by the Moray House Test (IQ) at age 11 years. One possible interpretation of our findings is that intellectual development and enculturation from age 11 years into adulthood has additional effects on lifestyle choices, including diet.

From a biological perspective, in order for dietary flavonoid intake to directly affect cognition, sufficient levels must be absorbed, metabolised and subsequently penetrate the blood-brain barrier. At the time of writing, no human bioavailability studies have been published. Data are available from small animal studies (rats/mice), which have demonstrated that flavonoids are rapidly metabolised to glucuronides, sulphates and O-methyl derivatives. Furthermore, flavanone, catechin and flavonol metabolites have been shown to cross the blood-brain barrier, having been found localised, in low concentrations in different regions of the brain $^{(4,36,37)}$. Although the evidence is limited, the potential action of flavonoids on cognitive function has been ascribed to their ability to modulate neuronal signalling, protect against neuronal losses and stimulate repair. Further studies involving humans are needed to provide more information in this area.

One limitation of the present study is that the measurements of diet were made at only one point in time, and therefore 
may not reflect long-term intake. It is not clear how much our dietary habits change over the years, and it may be that the subjects had significantly different flavonoid intakes in the past. Furthermore, if flavonoids do indeed affect cognitive function, it is not clear whether there is a cumulative effect over time, or whether the effects are more immediate. The present study would have been less likely to detect any possible links between flavonoid intake and cognition if the effects were more cumulative or related to previous flavonoid consumption. As such, any associations may have been attenuated. It should also be noted that this particular cohort would have had a different diet in childhood compared with children today, in view of factors such as post-war rationing of foods. We cannot be certain therefore that the results are fully generalisable to contemporary children. Furthermore, as with any cross-sectional study, it is not possible to determine the causal factor in any observed association. Random error in the measurement of dietary intake due to the use of FFQ is also likely, so we cannot rule out the possibility of type 2 errors due to the attenuation of associations as a result. Nonetheless, the repeatability of the FFQ has been shown to be generally good in a previous study ${ }^{(32)}$. Finally, participants with lower performance on the tests were under-represented in this analysis, as they were more likely to fail to complete the FFQ to the required standard. The likely effect of this is to attenuate true effect size. The results of the present study apply to differences in normal rather than pathological ageing and, therefore, cannot be extrapolated with confidence to those with impaired cognitive ability.

A major strength of the present study is the availability of data on IQ at age 11 years, which accounted for the largest proportion of the variance in test results at age 70 years. After allowing for this effect, the proportion of the variance associated with other socio-economic factors was generally low. Other strengths of the study include the detailed information on the participants' social background, the welldeveloped flavonoid database, the validation of the use of flavonoid intake estimates from the FFQ in older people and also the wide variety of cognitive tests which were administered. Based on an analysis of antioxidant and vitamin $\mathrm{B}$ intake and cognitive function in this cohort $^{(38)}$, the sample size had sufficient power to detect a standardised regression coefficient of about 0.07 in the fully adjusted model.

In summary, our findings do not support a causal role for flavonoids in the prevention of cognitive decline in later life. Instead, the authors suggest that future studies involving diet and cognitive function should include a marker of prior IQ as a confounding factor in the analysis. Childhood IQ is very useful in this regard, but the data are often not readily available. The NART has been shown to be a valid estimator of peak prior intelligence, and, as such, should ideally be included as an easy-to-measure potential confounding factor in future studies of this type.

\section{Acknowledgements}

The authors' contributions are as follows: C. B. performed the statistical analysis and data interpretation, and was involved in the writing and editing of the manuscript; J. K. and G. M. performed the statistical analysis, data interpretation, revisions to the manuscript and approval of the final version; J. C. and A. G. had a role in data collection, checking and management, revising the manuscript and approval of the final version; J. M. S. co-designed the statistical analysis models, revised the manuscript and approved the final version; I. J. D. designed the study, directed the study, trained and validated cognitive testing, co-designed the statistical analysis models, revised the manuscript and approved the final version. J. C. and A. J. G. are funded by the Sidney De Haan Award, part of the Disconnected Mind project funding from Help The Aged to I. J. D. and J. M. S. In addition, a Research Into Ageing programme grant to I. J. D. and J. M. S. also supported A. J. G. and J. C. Further funding was obtained from an SpR Start-up Grant from the British Geriatrics Society to C. B. We thank the Scottish Council for Research in Education for allowing access to the SMS1947. The present study was undertaken by the University of Edinburgh Centre for Cognitive Ageing and Cognitive Epidemiology, part of the cross-council Lifelong Health and Wellbeing Initiative. Funding from the BBSRC, EPSRC, ESRC and MRC is gratefully acknowledged. We thank the Lothian Birth Cohort 1936 study participants. We also acknowledge Lorna Aucott, Statistician from the University of Aberdeen, for her help with the statistical analysis. The authors declare that there are no conflicts of interest.

\section{References}

1. United Nations Human Development Report (2005) International cooperation at a crossroads: aid, trade and security in an unequal world.

2. Alzheimers UK (2009) Statistics. http://www.alzheimers. org.uk/site/scripts/documents_info.php?categoryID=200120\& documentID $=341$

3. Spencer JP, Vauzour D \& Rendeiro C (2009) Flavonoids and cognition: the molecular mechanisms underlying their behavioural effects. Arch Biochem Biophys 492, 1-9.

4. Spencer JP (2010) Beyond antioxidants: the cellular and molecular interactions of flavonoids and how these underpin their actions on the brain. Proc Nutr Soc 69, 244-260.

5. Crozier A, Jaganath IB \& Clifford MN (2009) Dietary phenolics: chemistry, bioavailability and effects on health. Nat Prod Rep 26, 1001-1043.

6. Kyle JAM \& Duthie GG (2006) Flavonoids in foods. In Flavonoids: Chemistry, Biochemistry and Applications, pp. 219-220 [OM Andersen and KR Markham, editors]. Boca Raton, FL: CRC Press.

7. Dajas F, Arredondo F, Echeverry C, et al. (2005) Flavonoids and the brain: evidences and putative mechanisms for a protective capacity. Curr Neuropharmacol 3, 193-205.

8. Francis ST, Head K, Morris PG, et al. (2006) The effect of flavanol-rich cocoa on the fMRI response to a cognitive task in healthy young people. J Cardiovasc Pharmacol 47, Suppl. 2, S215-S220.

9. Fisher NDL, Hughes M, Gerhard-Herman M, et al. (2003) Flavanol-rich cocoa induces nitric-oxide-dependent vasodilation in healthy humans. J Hypertens 21, 2281-2286.

10. Fisher NDL, Sorond FA \& Hollenberg NK (2006) Cocoa flavanols and brain perfusion. J Cardiovasc Pharmacol 47, Suppl. 2, S210-S214. 
11. Heiss C, Kleinbongard P, Dejam A, et al. (2005) Acute consumption of flavanol-rich cocoa and the reversal of endothelial dysfunction in smokers. J Am Coll Cardiol 46, 1276-1283.

12. Leikert JF, Rathel TR, Wohlfart P, et al. (2002) Red wine polyphenols enhance endothelial nitric oxide synthase expression and subsequent nitric oxide release from endothelial cells. Circulation 106, 1614-1617.

13. Middleton E Jr, Kandaswami C \& Theoharides TC (2000) The effects of plant flavonoids on mammalian cells: implications for inflammation, heart disease, and cancer. Pharmacol Rev 52, 673-751.

14. Scalbert A, Johnson IT \& Saltmarsh M (2005) Polyphenols: antioxidants and beyond. Am J Clin Nutr 81, Suppl. 1, 215S-217S.

15. Ramassamy C (2006) Emerging role of polyphenolic compounds in the treatment of neurodegenerative diseases: a review of their intracellular targets. Eur J Pharmacol 545, 51-64.

16. Balzer J, Heiss C, Schroeter H, et al. (2006) Flavanols and cardiovascular health: effects on the circulating NO pool in humans. J Cardiovasc Pharmacol 47, Suppl. 2, S122-S127.

17. Perez-Vizcaino F, Duarte J \& Andriantsitohaina R (2006) Endothelial function and cardiovascular disease: effects of quercetin and wine polyphenols. Free Radic Res 40, 1054-1065.

18. Spencer JPE, Vauzour D \& Rendeiro C (2009) Flavonoids and cognition: the molecular mechanisms underlying their behavioural effects. Arch Biochem Biophys 492, 1-9.

19. Commenges $\mathrm{D}$, Scotet $\mathrm{V}$, Renaud $\mathrm{S}$, et al. (2000) Intake of flavonoids and risk of dementia. Eur J Epidemiol 16, 357-363.

20. Engelhart MJ, Geerlings MI, Ruitenberg A, et al. (2002) Dietary intake of antioxidants and risk of Alzheimer disease. JAMA 287, 3223-3229.

21. Letenneur L, Proust-Lima C, Le Gouge A, et al. (2007) Flavonoid intake and cognitive decline over a 10 -year period. $\mathrm{Am}$ J Epidemiol 165, 1364-1371.

22. Nurk E, Refsum H, Drevon CA, et al. (2009) Intake of flavonoid-rich wine, tea, and chocolate by elderly men and women is associated with better cognitive test performance. J Nutr 139, 120-127.

23. Batty GD, Deary IJ, Schoon I, et al. (2007) Childhood mental ability in relation to food intake and physical activity in adulthood: the 1970 British Cohort Study. Pediatrics 119 e38-e45.

24. Akbaraly TN, Singh-Manoux A, Marmot MG, et al. (2009) Education attenuates the association between dietary pat- terns and cognition. Dement Geriatr Cogn Disord 27 , 147-154.

25. Corder EH, Saunders AM, Strittmatter WJ, et al. (1993) Gene dose of apolipoprotein E type 4 allele and the risk of Alzheimer's disease in late onset families. Science 261, 921-923.

26. Wisdom NM, Callahan JL \& Hawkins KA (2011) The effects of apolipoprotein $\mathrm{E}$ on non-impaired cognitive functioning: a meta-analysis. Neurobiol Aging 32, 63-74

27. Deary IJ, Gow AJ, Taylor MD, et al. (2007) The Lothian Birth Cohort 1936: a study to examine influences on cognitive ageing from age 11 to age 70 and beyond. BMC Geriatr 7, 28.

28. Office of Population Census and Surveys (1980) Classification of Occupations. London: HMSO.

29. Johnson W, Brett CE \& Deary IJ (2010) Intergenerational class mobility in Britain: a comparative look across three generations in the Lothian Birth Cohort 1936. Intelligence 38, 268-281.

30. Johnson W, Gow AJ, Corley J, et al. (2010) Location in cognitive and residential space at age 70 reflects a lifelong trait over parental and environmental circumstances: The Lothian Birth Cohort 1936. Intelligence 38, 402-411.

31. Willet WC (1998) Nutritional Epidemiology, 2nd ed. New York: Oxford University Press.

32. Jia X, Craig LCA, Aucott LS, et al. (2008) Repeatability and validity of a food frequency questionnaire in free-living older people in relation to cognitive function. $J$ Nutr Health Aging 12, 735-741.

33. Luciano M, Gow AJ, Harris SE, et al. (2009) Cognitive ability at age 11 and 70 years, information processing speed, and APOE variation: The Lothian Birth Cohort 1936 Study. Psychol Aging 24, 129-138.

34. Corley J, Jia X, Kyle JA, et al. (2010) Caffeine consumption and cognitive function at age 70: The Lothian Birth Cohort 1936 Study. Psychosom Med 72, 206-214.

35. Deary IJ, Whalley LJ, Lemmon H, et al. (2000) The stability of individual differences in mental ability from childhood to old age: follow-up of the 1932 Scottish Mental Survey. Intelligence 28, 49-55.

36. Youdim KA, Shukitt-Hale B \& Joseph JA (2004) Flavonoids and the brain: interactions at the blood-brain barrier and their physiological effects on the central nervous system. Free Radic Biol Med 37, 1683-1693.

37. Youdim KA, Qaiser MZ, Begley DJ, et al. (2004) Flavonoid permeability across an in situ model of the blood-brain barrier. Free Radic Biol Med 36, 592-604.

38. McNeill G, Jia X, Whalley LJ, et al. (2011) Antioxidant and B vitamin intake in relation to cognitive function in later life in the Lothian Birth Cohort 1936. Eur J Clin Nutr (In the Press). 